\title{
The percutaneous rotary lithotrite: a new approach to the treatment of symptomatic cholecystolithiasis
}

\author{
A Gillams, J J Donald, R C G Russell, A R W Hatfield, W R Lees
}

\begin{abstract}
This report evaluates the use of a new device for destruction of gall stones, the KenseyNash Lithotrite (Baxter Corporation, California, USA). The principle of the instrument is that of a liquidiser with an impeller that emulsifies stones. Twenty five patients were treated; 13 patients were considered unfit for conventional treatment (complex group) and 12 elected to have the procedure (non-complex group). In the complex group nine patients were treated under local anaesthesia. Only six of the 13 patients had a clear gall bladder at the end of the first procedure, but after further treatments that included cholecystoscopy, endoscopic sphincterotomy, and percutaneous cholecystolithotomy 11 patients had a gall bladder free of stones. The morbidity was high, mainly due to pain and bile leaks, causing prolonged stays in hospital (median 18 days). In the non-complex group six patients had the procedure performed under local anaesthesia. Ten patients had a successful clearance of the gall bladder, and the remaining two patients had the stones removed at cholecystoscopy. Despite good clearance, the morbidity was high, with eight emergency admissions on account of complications and a prolonged duration of stay (median 13 days). In conclusion the technique is effective, but the morbidity is high. Further development is required if this technique is to be included in the alternative treatments for the management of gall stones.
\end{abstract}

(Gut 1993; 34: 837-842)

The introduction of laparoscopic cholecystectomy has changed the approach to the treatment of symptomatic gall stones. There remains a group of patients, however, who are either unsuitable for general anaesthesia because of medical contraindications or who wish to conserve their gall bladders. ${ }^{1}$ Existing conservative treatments all have their limitations. ${ }^{2}$ This report evaluates the use of a new device, the KenseyNash Lithotrite, for gall stone destruction. The principle is that of a liquidiser; a rapidly rotating impeller $(30000 \mathrm{rpm})$ within a confined space filled with a liquid creates a vortex that sucks solid material on to the impeller, which pulverises the solid. A fine mud is produced, and this is aspirated from the gall bladder. The apparatus is introduced into the gall bladder through a small (12 French) percutaneous tract under local anaesthesia and intravenous sedation/analgesia. A prototype has been used successfully on human gall stones introduced into swine gall bladders. ${ }^{34}$ Initial clinical results have been reported for 10 patients. The procedure was successful in nine; complications included bile leakage, and one patient developed phlegmonous cholecystitis necessitating cholecystectomy. ${ }^{5}$ At three to six weeks after the procedure stones or fragment aggregates were present in six patients. At follow up (median of 28 (range six to 52) weeks) three patients had fragment aggregates and one patient an intact calculus. It was considered that many of these problems were related to inexperience in the management of stones within a retained gall bladder. As a result of the considerable experience with percutaneous cholecystolithotomy, ${ }^{67}$ it was thought that these problems could be rapidly solved and the technique established to replace percutaneous cholecystolithotomy as it could be performed under local anaesthesia by the transhepatic route and a postprocedural drain could be avoided. Our initial experience is described.

\section{Patients}

Twenty five patients who were referred on account of characteristic biliary symptoms and were known to have gall stones on the basis of previous ultrasound examinations were accepted for treatment by the rotary lithotrite. Thirteen patients were referred as they had been considered unsuitable for conventional cholecystectomy on account of various coexistent medical diseases (complex group, Table I), and 12 patients were referred as they had requested minimally invasive treatment for their gall bladder disease or specifically wished their gall bladder to be preserved (non-complex group, Table II). A detailed ultrasound assessment was undertaken before the procedure to determine: (a) the position of the gall bladder to ensure that it was suitable for safe percutaneous puncture; (b) the size and stone load of the gall bladder to ensure that there was adequate working space for the lithotrite and that a vortex could be achieved; and $(c)$ the function of the gall bladder by use of a standard fat stimulus. Contraindications to the procedure included stones greater than $3 \mathrm{~cm}$ in diameter or limited working space within the gall bladder, either because of a small volume $(<8 \mathrm{ml})$ or a large stone load $(>75 \%$ of gall bladder volume). Gall bladders that contracted after a fatty stimulus were preferred, as previous experience suggests that stasis is likely to lead to recurrent stone formation. ${ }^{7}$ The median number of stones treated was three (range one to 20) with a median size of $9 \mathrm{~mm}$ (range five- $28 \mathrm{~mm}$ ). Gall bladder contractility was present in 16 patients before the procedure.

Patients were admitted the evening before the procedure with the plan of discharging the patient on the second or third day after the procedure with the drain from the gall bladder 
TABLE I Complex group

\begin{tabular}{|c|c|c|c|c|c|c|c|c|c|c|}
\hline Patient & Sex & $\begin{array}{c}\text { Age } \\
(y r)\end{array}$ & $\begin{array}{l}\text { Weight } \\
(\mathrm{kg})\end{array}$ & $\begin{array}{l}\text { ASA } \\
\text { grade }\end{array}$ & Anaesthesia & $\begin{array}{l}\text { Rotation } \\
\text { time } \\
\text { (min) }\end{array}$ & $\begin{array}{l}\text { Stone } \\
\text { clearance }\end{array}$ & $\begin{array}{l}\text { No of } \\
\text { admissions }\end{array}$ & $\begin{array}{l}\text { Duration } \\
\text { of stay } \\
\text { (days) }\end{array}$ & Complications \\
\hline I & $\mathrm{F}$ & 52 & 53 & 2 & General & 7 & Yes & 2 & 8 & Emergency readmission with pain; asparate transaminase $\uparrow$ \\
\hline II & $\mathbf{F}$ & 88 & 43 & 3 & $\begin{array}{l}\text { Local } \\
\text { Local }\end{array}$ & $\begin{array}{l}30 \\
12\end{array}$ & $\begin{array}{l}\text { No } \\
\text { No }\end{array}$ & 4 & 22 & $\begin{array}{l}\text { Initial failed clearance; repeat procedure; eventual clearance by } \\
\text { cholecystoscopy }\end{array}$ \\
\hline III & $\mathrm{F}$ & 79 & 70 & 2 & $\begin{array}{l}\text { Local } \\
\text { Local }\end{array}$ & 30 & $\begin{array}{l}\text { No } \\
\text { No }\end{array}$ & 2 & 18 & $\begin{array}{l}\text { Pleural effusion and atrial fibrillation after lst procedure; repeat } \\
\text { procedure: prong lost; stone fragments and prong retrieved at } \\
\text { cholecystoscopy }\end{array}$ \\
\hline IV & $\mathrm{F}$ & 71 & 77 & 2 & Local & 8 & No & 3 & 9 & Stones noted on tubogram; track lost at cholecystoscopy \\
\hline V & $\mathbf{F}$ & 52 & 96 & 2 & General & 47 & Yes & 2 & 6 & Severe pain after lithotrite \\
\hline VI & $\mathbf{F}$ & 76 & 72 & 3 & General & 17 & Yes & 1 & 14 & Severe medical problems and pain \\
\hline VII & $\mathbf{F}$ & 78 & 58 & 4 & Local & 8 & Yes & 2 & 13 & $\begin{array}{l}\text { Stone in common bile duct pre-lithotrite - ERCP and sphincterotomy; } \\
\text { successful lithotrite - no complications }\end{array}$ \\
\hline VIII & $\mathrm{F}$ & 75 & 83 & 3 & $\begin{array}{l}\text { Local } \\
\text { Local }\end{array}$ & 2,1 & $\begin{array}{l}\text { No } \\
\text { No }\end{array}$ & 3 & 34 & $\begin{array}{l}\text { lst stage; incomplete clearance; failed cholecystoscopy; } \\
\text { 2nd stage; catheter displaced; incomplete clearance; unsuccessful } \\
\text { cholecystoscopy; }\end{array}$ \\
\hline IX & & & & & Local & $\bar{c}$ & Yes & & & 3rd stage; complete clearance \\
\hline $\mathbf{X}$ & $M$ & 74 & 81 & 3 & Local & 15 & Yes & 3 & 8 & Readmitted after tube removal with severe pain \\
\hline $\mathbf{X}$ & 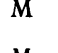 & 53 & 73 & 3 & Local & 52 & Yes & 1 & 21 & $\begin{array}{l}\text { Retained prong: removed at cholecystoscopy; exacerbation cardiac } \\
\text { failure }\end{array}$ \\
\hline XI & $\mathbf{M}$ & 70 & 70 & 2 & General & 8 & No & 4 & 19 & $\begin{array}{l}\text { Retained stones: removed at cholecystoscopy; stones in duct: ERCP and } \\
\text { sphincterotomy }\end{array}$ \\
\hline XII & $\mathrm{F}$ & 83 & 61 & 4 & $\begin{array}{l}\text { Local } \\
\text { Local }\end{array}$ & $\begin{array}{r}37 \\
4\end{array}$ & No & 1 & 27 & $\begin{array}{l}\text { Staged clearance: stone lodged in cystic duct; severe medical problems } \\
\text { prevented further endoscopy } 11 / 12 \text { days later; laparoscopic } \\
\text { cholecystectomy }\end{array}$ \\
\hline XIII & F & 86 & 63 & 2 & $\begin{array}{l}\text { Local } \\
\text { Local }\end{array}$ & $\begin{array}{r}8 \\
34\end{array}$ & No & 2 & 33 & $\begin{array}{l}\text { Readmitted with severe pain; failed lithotrite; successful percutaneous } \\
\text { cholecystolithotomy }\end{array}$ \\
\hline
\end{tabular}

$\mathrm{ERCP}=$ endoscopic retrograde cholangiopanreatographic examination.

TABLE II Non-complex group

\begin{tabular}{|c|c|c|c|c|c|c|c|c|c|c|}
\hline Patient & Sex & $\begin{array}{l}\text { Age } \\
(y r)\end{array}$ & $\begin{array}{l}\text { Weight } \\
(k g)\end{array}$ & $\begin{array}{l}\text { ASA } \\
\text { grade }\end{array}$ & Anaesthesia & $\begin{array}{l}\text { Rotation } \\
\text { time } \\
(\text { min })\end{array}$ & $\begin{array}{l}\text { Stone } \\
\text { clearance }\end{array}$ & $\begin{array}{l}\text { No of } \\
\text { admissions }\end{array}$ & $\begin{array}{l}\text { Duration } \\
\text { of stay } \\
\text { (days) }\end{array}$ & Complications \\
\hline I & $\mathbf{F}$ & 63 & 62 & 1 & General & 10 & Yes & 2 & 24 & Bile leak after tubogram; subphrenic collection and pleural effusion \\
\hline II & $\mathbf{F}$ & 38 & 81 & 1 & Local & 9 & Yes & 2 & 8 & Bile leak; severe pain \\
\hline III & M & 71 & 62 & l & Local & 12 & Yes & 3 & 9 & Bile leak; subphrenic collection \\
\hline IV & $\mathbf{M}$ & 72 & - & 1 & Local & 10 & No & 2 & - & Stones cleared at cholecystoscopy \\
\hline $\mathrm{V}$ & M & 77 & 65 & 1 & General & 15 & No & 3 & 16 & Stones cleared at 2 nd cholecystoscopy with electrohydraulic lithotripsy \\
\hline VI & M & 75 & 88 & 2 & Local & 14 & Yes & 2 & 8 & Pyrexial postoperatively; treated with antibiotic \\
\hline VII & $M$ & 75 & 60 & 1 & General & 25 & Yes & 2 & 20 & $\begin{array}{l}\text { Slow recovery due to pain; no collection seen on ultrasound scan. } \\
\text { Emergency admission with pain; cholecystectomy }\end{array}$ \\
\hline VIII & $M$ & 74 & 91 & 2 & Local & 52 & Yes & 2 & 6. & Uneventful course \\
\hline IX & M & 44 & 79 & 1 & General & 11 & Yes & 2 & 3 & Uneventful course; one episode severe pain \\
\hline $\mathrm{X}$ & $\mathrm{F}$ & 33 & 62 & 1 & General & 11 & Yes & 3 & 18 & Bile leak requiring percutaneous drainage \\
\hline XI & $\mathrm{F}$ & 34 & 50 & 1 & General & 30 & Yes & 3 & 22 & Bile leak requiring percutaneous drainage \\
\hline XII & $\mathbf{F}$ & 49 & 57 & 1 & Local & 21 & Yes & 2 & 13 & Bile leak after cholecystoscopy; managed by endoscopic sphincterotomy \\
\hline
\end{tabular}

either spigotted or, if pain occurred, discharging into a bag as in the procedure of percutaneous cholecystolithotomy. The patient returned 10 to 12 days after the procedure for a tubogram to assess the presence or absence of stones in the gall bladder and the patency of the cystic and common bile ducts. The patient was discharged the day after. All patients received ursodeoxycholic acid (5 mg/kg/day) and chenodeoxycholic acid ( $7 \mathrm{mg} / \mathrm{kg} /$ day) for a period of at least three months after the procedure as previous experience with percutaneous cholecystolithotripsy had suggested that this helped to remove the fragments noted at the review examination. Patients were followed up for six months with ultrasound and assessment of symptoms.

The rotary lithotrite consists of a central rotating metal impeller surrounded by a basket of metal prongs mounted on a catheter (Fig 1). Rotation of the impeller to speeds of $30000 \mathrm{rpm}$ generates a vortex that sucks the gall stones into the basket, on to the impeller where the stones are reduced to a fine sediment (Fig 2). The metal basket holds the gall bladder mucosa clear of the impeller. The whole apparatus is collapsed down for introduction into and removal from the gall bladder via a 12 French introducer sheath.

The procedure is performed with the patient supine on a fluoroscopy table with continuous blood pressure, cardiac, and oximetry monitoring. Prophylactic intravenous antibiotics $(8 \mathrm{~g}$ mezlocillin daily) were given for 24 hours. Patients received either a general anaesthetic or intravenous sedation (diazemuls), analgesic (pethidine), and local anaesthetic $(20 \mathrm{ml} 2 \%$ lignocaine) injected subcutaneously and along the percutaneous tract. Initially the gall bladder was opacified with contrast (half strength Conray 280) injected through a $22 \mathrm{G}$ spinal needle introduced into the gall bladder under ultrasound guidance. With a combination of ultrasound and fluoroscopic guidance the definitive puncture with an $18 \mathrm{G}$ longdwell needle (BectonDickinson) was performed. Either a transhepatic, subhepatic, or transperitoneal route could be used. The transhepatic route was used in 15 and the transperitoneal in 10 patients. A 0.035 inch Lunderquist wire with a $3.5 \mathrm{~cm}$ floppy tip (Wilson Cook, Europe) was introduced through the longdwell sheath and the tip positioned in the proximal cystic duct. The percutaneous tract was dilated with 6-8 French Teflon dilators (Wilson Cook, Europe) and then 9-12 French telescopic metal dilators (Olympus, Keymed) introduced in a coaxial fashion. ${ }^{7}$ The dilators were removed and the introducer sheath inserted into the gall bladder and secured by inflating a small $(1 \mathrm{ml})$ balloon or by deploying a helical plastic anchor. After removal of the guidewire the lithotrite was introduced in its collapsed form through the sheath and once within the gall bladder the lithotrite basket was 

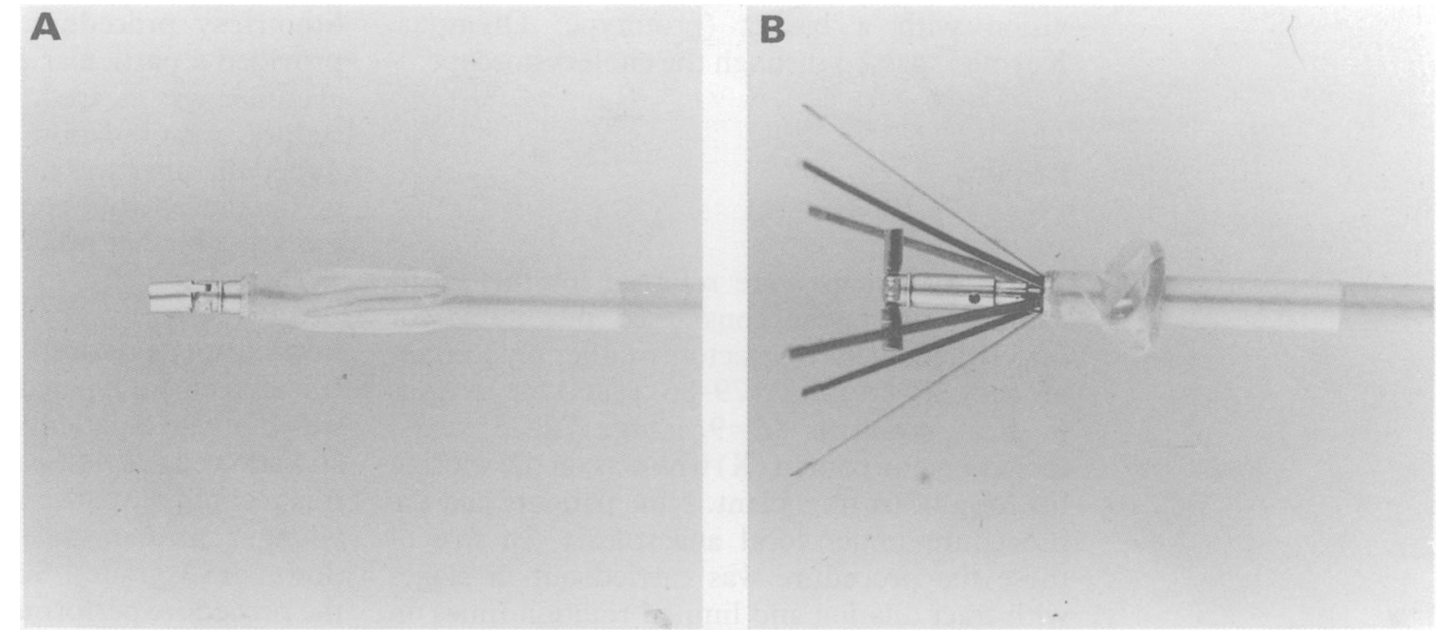

Figure 1: Working end of the lithotrite catheter $(A)$ closed for introduction into the gall bladder and $(B)$ open showing the impeller and basket of metal prongs.

opened and locked in the open position. Before starting rotation of the impeller the lithotrite was gently rotated under fluoroscopy to ensure that it was free of the gall bladder wall.

Rotation of the impeller was commenced at $5000 \mathrm{rpm}$ and gradually increased to a maximum of $30000 \mathrm{rpm}$. Continuous saline infusion through a side port of the lithotrite was maintained throughout to keep the gall bladder distended. The procedure was monitored by fluoroscopy. Ultrasound monitoring was not possible as impeller rotation generated multiple tiny bubbles that produced a diffuse intraluminal echogenicity.

Initially the impact of stones on the impeller was palpable by the operator, but as stones were reduced to a fine sediment the impacts ceased. Rotation was continued until the gall bladder was clear on fluoroscopy and no impacts had

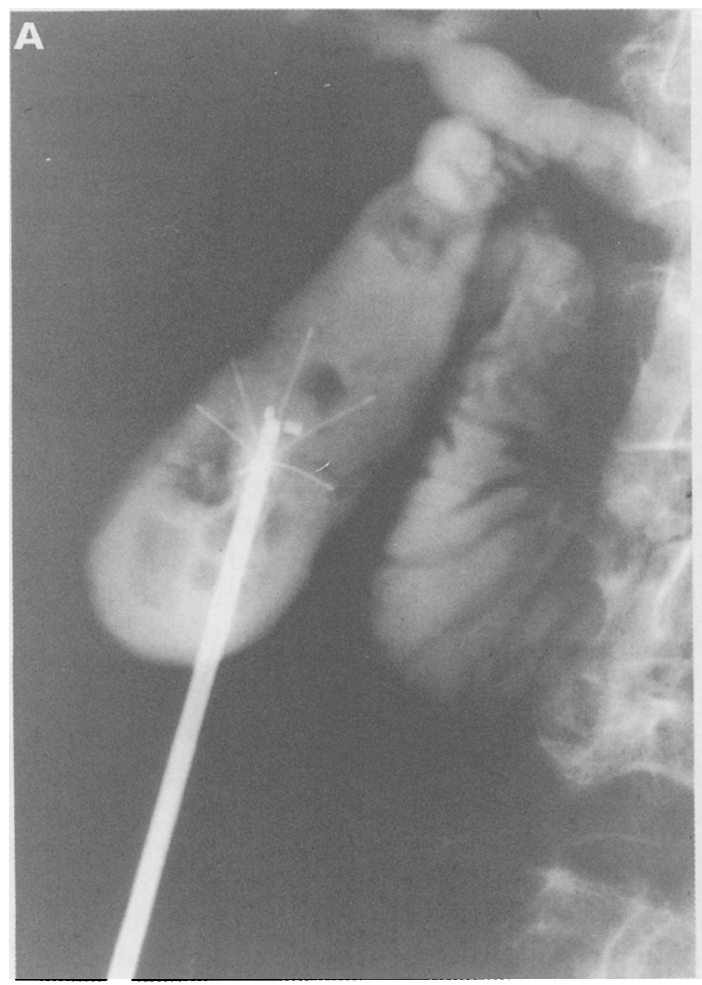

been felt for five minutes. As much sediment as possible was then aspirated. At the end of the procedure a 10 or 12 French Cope loop drainage catheter (Cook Urological) was left in the gall bladder on free drainage.

At the time of the return visit, a cholecystoscopy was performed with a 10 or 12 French prototype flexible cholecystoscope (Olympus, $\mathrm{XCHF}$, Keymed) with a 3 French working channel. This procedure was undertaken by passing a guidewire down the drainage catheter under radiological control. The drain was then removed and the cholecystoscope passed over the guidewire into the lumen of the gall bladder. Provided no stones were found and the patency of the ducts was confirmed with contrast studies, the cholecystoscope was withdrawn and the tract allowed to close. If stones were found at cholecystoscopy, the stones were removed under

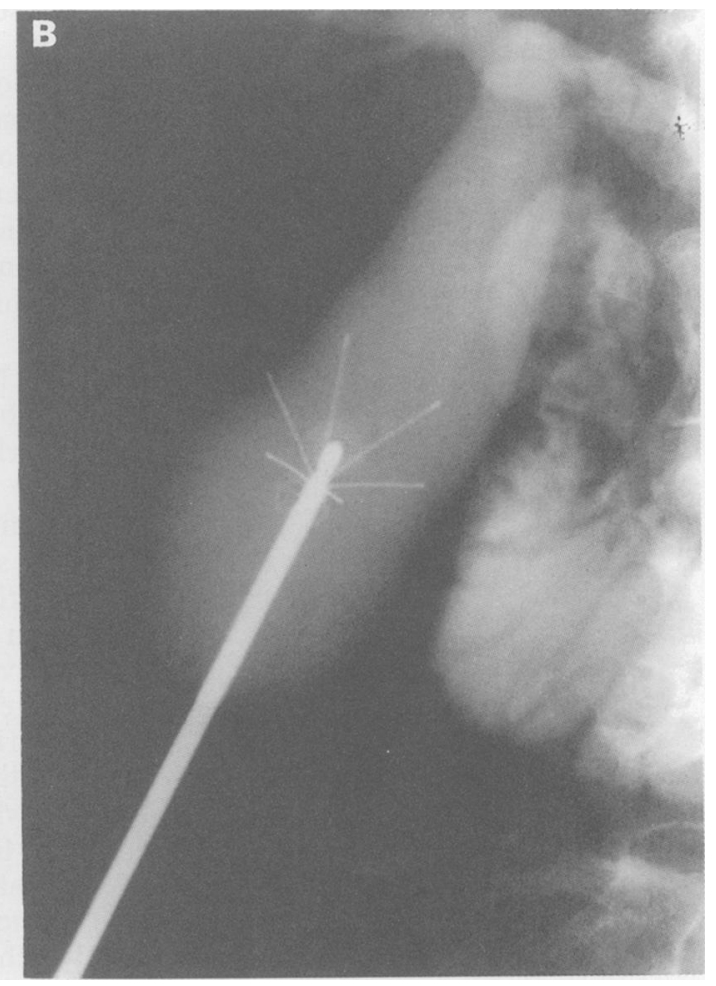

Figure 2: (A) The lithotrite deployed within the gall bladder; a stone is held within the basket. (B) After rotation of the impeller the gall bladder has been cleared of stones. 
vision with a basket (prototype: Olympus, Keymed) passed through the cholecystoscope.

\section{Results}

\section{COMPLEX GROUP}

Thirteen patients were referred for this treatment as they were considered unsuitable for conventional cholecystectomy either on account of age $(n=4$, range $79-86$ years) or serious medical problems $(n=9$, range $72-78$ years) including one patient $(\mathrm{X})$ who was on the waiting list for a heart transplant. Nine patients had the procedure under local anaesthesia. In five of these the procedure was carried out in stages with tract dilation and limited rotation times on account of local discomfort. There was considerable variation in the tolerance to the procedure with one patient able to accept a 52 minute rotation time. Only six of the 13 patients had a clear gall bladder at the end of the first procedure. One patient had stones cleared at a third stage, two patients had the remaining fragments removed at cholecystoscopy, and one patient by percutaneous cholecystolithotomy. One patient had a combined cholecystoscopy and endoscopic sphincterotomy to clear stones from the gall bladder and bile duct, leaving two patients with a failed clearance, one of whom is without symptoms (patient IV). The other had continued pain that was eventually successfully managed by a laparoscopic cholecystectomy. The ideal inpatient stay was two admissions of three and two days; no patient achieved this ideal, with the patient nearest spending four days in hospital on the first occasion, the delay in discharge being due to severe pain. The median number of admissions was two (range one to four) and the median duration of stay was 18 (range six to 34) days. No patient in this group could be considered to have had a successful and uneventful procedure. The major problem was that these were ill patients in whom the slightest disturbance caused a major exacerbation of their underlying medical condition. The most common problem was pain, often due to subhepatic bile collections, or difficulties with track or drainage tubes. Particular technical problems were the fracture and retention of a prong in two patients, presumably due to the prong impinging on the impeller. Skilled cholecystoscopy (ARH) retrieved the situation by extracting the prong in both cases. A particularly difficult problem is the passage of fragments down the cystic duct; provided these fragments pass into the common bile duct endoscopic sphincterotomy successfully manages the situation (patient VII, XI), but if the stone remains in the cystic duct removal can be impossible as even the smallest scope or basket will not dislodge the stone (patient XII). One patient (patient VIII), however, had a stone successfully manipulated from the cystic duct into the gall bladder where the stone was fragmented at subsequent lithotripsy. A large stone can provide a technical problem particularly if hard; in patient XIII the lithotrite cored a hole in a $28 \mathrm{~mm}$ stone, which did not break up and required subsequent cholecystoscopy and contact lithotripsy followed by a second rotary lithotripsy procedure. A septate gall bladder provided a particular problem in that the lower chamber was cleared, but despite a repeat procedure, manipulation of the lithotrite into the upper chamber was not accomplished; clearance was achieved by percutaneous cholecystolithotomy as a further procedure (patient XIII).

\section{NON-COMPLEX GROUP}

It had been anticipated that this group of fit and well motivated patients would have fared better, and allowed full assessment of the advantages of this technique, particularly as the patients were younger, median age 63 (range 33-77) and fitter (lower ASA grade). Six patients agreed to have the procedure performed under local anaesthesia and six opted for general anaesthesia. As they were fitter all had the procedure in one stage and withstood up to 52 minutes rotation time (patient VIII). Ten of the 12 patients had a successful clearance of the gall bladder, with the procedure described in all these patients as technically very easy. Both patients who had stones retained at the end of the procedure had satisfactory clearance at cholecystoscopy. The median number of admissions was two, but in this group of patients, there were eight emergency admissions on account of complications, accounting for the prolonged duration of stay (median 13 (range three to 24) days). The cause of the emergency admissions and prolonged stay was not due to the procedure itself, but due to pain, which was invariably related to bile leaks that occurred late in the course of the recovery. Initially these were missed on ultrasound, and only shown on careful computed tomography scanning as the fluid was not localised in the right upper quadrant. Most resolved with simple conservative treatment and antibiotics but some required the insertion of percutaneous drains (patient X, XI) or simple aspiration (patients I and III). Patient XII was managed by an endoscopic sphincterotomy after percutaneous drainage of the collection. One patient was admitted to another hospital (patient VII), and despite good conservative management failed to resolve rapidly. Thus the surgeon, after discussion, decided to undertake cholecystectomy and confirmed a gall bladder perforation; this patient subsequently did well.

\section{PERCUTANEOUS FLEXIBLE CHOLECYSTOSCOPY}

Percutaneous flexible cholecystoscopy was performed on 19 patients on or after the 10th postprocedural day. The gall bladder mucosa seemed normal in 16 patients; in two there was evidence of chronic inflammation, and in one patient the gall bladder was acutely inflammed. In no patient was there evidence of perforation, even in those in whom bile collections were found. Of particular note was the therapeutic value of the cholecystoscopy: in the complex group two prongs and several stones were removed (complete clearance in patient II), and in the non-complex group complete clearance was achieved in two patients (IV and V).

Follow-up data are available in 18 patients between three and six months after the procedure. Eleven patients have remained free of 
stones throughout; two patients with fragments noted at three months were clear at six months after continued oral bile acid treatment. Four patients have developed recurrent stones, one of whom has had a laparoscopic cholecystectomy, a second who is about to have a laparoscopic procedure, and two who are free of symptoms. The 18th patient is symptom free after the cholecystectomy performed for a bile leak. Gall bladder contractility was present in nine of the 10 patients at follow up; in two of these patients a non-contractile gall bladder had become contractile and in one patient, gall bladder contractility had been lost. The remaining six patients are free of symptoms, but have not attended for ultrasound examination. The seventh patient (patient X: complex group) died on the 60th postprocedural day on account of a further exacerbation of his cardiac failure.

\section{Discussion}

No new procedure is without technical problems, which account for the phenomenon of the learning curve that occurs with all new technical treatments. The importance of assessment, after it has been established that the technique achieves what it has set out to do (phase I), is to determine that the technique is safe, effective, and worthy of further trial (phase II). Of importance at this stage is whether the technique merits wider and detailed comparative trials or is so good that such trials are not justified on ethical considerations or is so good that patient pressure is such that carefully controlled trials cannot be mounted (phase III). The objective of this report is to assess whether an experience with 25 patients justifies further development of the apparatus, and a move to a phase III trial.

The experience reported suggests that the method is effective in that at the end of the rotary lithotrite procedures 17 patients had a gall bladder clear of stones, and with subsequent procedures such as cholecystoscopy, endoscopic sphincterotomy, and percutaneous cholecystolithotomy all but two patients had stones cleared from the biliary system. Nevertheless, this was only achieved as a consequence of complex and sophisticated technology inappropriate to the management of a common condition for which there are simpler alternative treatments. For instance, the small diameter flexible cholecystoscopes are still at an early stage of development giving limited views of the gall bladder, and require experience to manipulate and perform direct contact lithotripsy. Retrieval of stones, fragments, or prongs is difficult as the working channel within the scope is narrow and allows only limited instrumentation.

The complications, which occurred in all patients apart from two who achieved the protocol, could be overcome, and no early deaths were recorded in a high risk group of patients, but pain and bile leakage were major problems. The pain was difficult to understand, but could be severe, occurring in the absence of obvious leakage of bile or other complication. The cause of the pain was attributed to gall bladder irritability, as it resembled the patients' original attacks of biliary colic; yet on cholecystoscopy at or after $\mathbf{1 0}$ days only one patient had appreciable mucosal inflammatory change. Nevertheless, microscopic examination of the gall bladder does show significant submucosal disruption (Cuschieri A, personal communication). The high incidence of biliary leakage could not be easily explained. The transhepatic route was used in 15 patients and the transperitoneal route in 10 patients. The first was used initially to prevent leakage, but was abandoned in favour of the transperitoneal route to achieve a better vortex and easier stone fragmentation. Leakage occurred with equal frequency in both groups. It is possible that this leakage was caused by minute perforations of the gall bladder allowing transudation of bile, that healed by the time of cholecystoscopy. Certainly the surgeon who performed the cholecystectomy on day 12 after the procedure was sure that he had found a perforation in the gall bladder separate from the puncture site. A second cause for the leakage was misadventure with the tract from the skin to the gall bladder. Modern siliconised tubes are less irritant than the old rubber catheters with the consequence that the walling off of the tract from the peritoneal cavity by fibrous tissue is less effective and more prone to leakage. To have left the tube and drain in place for 14-21 days would have been ideal but less acceptable to the patient. Unfortunately, no effective gall bladder plug has been developed despite considerable research in this field.

Our experience with percutaneous cholecystolithotomy followed an identical learning curve ${ }^{6}$; it was not until the first 50 procedures had been performed that a routine was established and the technical problems overcome. For instance access to the gall bladder was lost in one patient, a well described problem ${ }^{10}$ and the absence of a safety guide wire meant that greater care with the track had to be exercised. Difficulties determining whether or not the gall bladder was clear of stones were encountered; indeed, four patients were thought to be free of stones at the end of the procedure only to have fragments found on the 10th day. Fluoroscopy in these circumstances has been previously reported to be inaccurate, detection of impacts on the lithotrite also proved to be inaccurate, and ultrasound immediately after the procedure was non-contributory, showing diffuse echogenicity throughout the gall bladder lumen. This appearance has been explained by multiple tiny bubbles produced by rotation of the impeller. For this reason cholecystoscopy seems likely to be a permanent part of this procedure as it is necessary to confirm gall bladder clearance and to remove very small stones left behind after the lithotrite procedure.

The objective of a phase II type study is to determine whether this procedure is a useful addition to those already available for treating gall stones. The answer to this question lies in the complex problem of whether there is ever a role for retaining the gall bladder in the presence of stones, ' and whether it is the gall bladder that is the cause of the stones such that recurrence of stones is inevitable. ${ }^{12}$ On the other hand, the loss of the gall bladder reservoir, the increase in gastro-oesophageal reflux, and the possible relation with colonic neoplasia suggest that 
young patients who develop gall stones in relation to a particular episode - for example, pregnancy - may be better served by preservation of the gall bladder. The other group in whom gall bladder preservation is preferable are the old, the ill, and the high risk patients such as those described in the complex group. Patients presenting with an acute cholecystitis can be treated with immediate improvement by percutaneous drainage. To complete the procedure at a later date under local anaesthesia seems appropriate and in line with the modern concepts of minimally invasive surgery.

If it is accepted that there is a role, though admittedly limited, for managing gall stone disease by preservation of the gall bladder, should the rotary lithotrite be the method of choice? Technically it has an advantage over percutaneous cholecystolithotomy in that it requires a tract only 12 French in diameter as opposed to 30 French. ${ }^{6}$ The use of dissolution agents such as methyl-t-butylether or similar substances with or without computer controlled pumps have similar problems to those encountered in this study. Thus the rotary lithotrite could have a role in the management of gall stones if the problems encountered can be overcome.

To overcome these problems, further experience and development is necessary. The company involved (Baxter Corporation, California, USA) chose to test the instrument in multiple centres, such that no centre gained great experience and all encountered problems similar to those described in this paper. Problems relating to the instrument were rapidly overcome. After prongs were retained in two patients modifications were made to the instrument. Other modifications were made to simplify insertion and use of the machine. Yet, problems related to patients could not be modified, and undoubtedly further study is required to define for how long and at what speed the impeller can be rotated without microscopic damage to the gall bladder in vivo. Studies are required to determine the best drain- age catheter to use after the procedure and how long that catheter should be left in situ to provide easy and safe access for the cholecystoscope. The hope is that an outpatient procedure under local anaesthesia might be achieved with this procedure, with preservation of the gall bladder. Such an outcome would require continued development, which in terms of the return on the capital investment would either mean an instrument too expensive to use or the absence of a treatment modality.

In conclusion, this study shows that the rotary lithotrite achieves stone emulsification with clearance of stones from the gall bladder, but much further work is necessary to overcome the complications found in the study before a true assessment of the technique can be made.

Our thanks are due to Baxter Corporation, California, USA, who provided the rotary lithotrite and Keymed and Olympus who provided the rotary lithotrite
provided the cholecystoscopes.

1 Walsh TN, Russell RCG. Cholecystectomy and gallbladder conservation. Br F Surg 1992; 79: 4-5.

Cheslyn-Curtis S, Russell RC. New trends in gallstone management. Brf Surg 1991; 78: 143-9.

3 Miller FJ, Kensey KR, Nash JE. Experimental percutaneous gallstone lithotripsy: results in swine. Radiology 1989; 170 985.

4 Dempsey D, Contreras C, Milner R, Abrahams C, White J. Percutaneous ablation of gallstones. F Surg Res $1990 ; 49$ 116-20.

5 Miller F, Rose S, Buchi K, Hunter J, Nash J, Kensey K. Percutaneous rotational contact biliary lithotripsy: initial results with the Kensey-Nash Lithotrite. Radiology 1991; 178: 781-5.

6 Chiverton SG, Inglis JA, Judd C, Kellett MJ, Russell RC Wickham JE. Percutaneous cholecystolithotomy: the first 60 patients. BMF 1990; 300: 1310-2.

7 Cheslyn-Curtis S, Gillams A, Russell RCG, Donald J, Lake SP, Hatfield ARW, Lees WR. Selection, management and early outcome of 113 patients with symptomatic gallstones treated by percutaneous cholecystolithotomy. Gut 1992; 33 1253-9.

8 Neugebauer E, Troidl H, Spangenberger W, Dietrich A Lefering $R$, and the Cholecystectomy Study Group. Conventional versus laparoscopic cholecystectomy and the randomised controlled trial. BrFS urg 1991; 78: 150-4.

9 Cope C, Burke D, Meranze SG. Percutaneous extraction of gallstones in 20 patients. Radiology 1990; 176: 19-24.

10 Kellett M, Russell RCG, Wickham JE. Percutaneous cholecystolithotomy. Endoscopy 1989; 21: 365-6.

11 Spiro HM. Diagnostic laparoscopic cholecystectomy. Lancet 1992; 339: 167-8.

12 O'Donnell LJD, Wilson P, Guest P, Catnach SM, McLean A, Wickham JEA, et al. Indomethacin and postprandial gallbladder emptying. Lancet 1992; 339: 269-71. 